\title{
Antitubercular Activity and Inhibitory Effect on Epstein-Barr Virus Activation of Sterols and Polyisoprenepolyols from an Edible Mushroom, Hypsizigus marmoreus
}

\author{
Toshihiro Akihisa, ${ }^{*, a}$ Scott Gary Franzblau, ${ }^{b}$ Harukuni Tokuda, ${ }^{c}$ Masaaki Tagata, ${ }^{a}$ \\ Motohiko Ukiya, ${ }^{a}$ Tsunetomo Matsuzawa, ${ }^{d}$ Koichi Metori, ${ }^{e}$ Yumiko Kimura, ${ }^{e}$ \\ Takashi SUZUKI, ${ }^{e}$ and Ken YASUKAWA ${ }^{e}$ \\ ${ }^{a}$ College of Science and Technology, Nihon University; 1-8 Kanda Surugadai, Chiyoda-ku, Tokyo 101-8308, Japan: \\ ${ }^{b}$ Institute for Tuberculosis Research, College of Pharmacy (M/C 964), University of Illinois at Chicago; 833 S. Wood St., \\ Chicago, IL 60612, U.S.A.: ${ }^{c}$ Department of Biochemistry and Molecular Biology, Kyoto Prefectural University of \\ Medicine; Kyoto 602-0841, Japan: 'Agricultural Technology Institute of Nagano Farmers' Federation; 787-1 Suzaka, \\ Sazaka, Nagano 382-0084, Japan: and ${ }^{e}$ College of Pharmacy, Nihon University; 7-7-1 Narashinodai, Funabashi, Chiba \\ 274-8555, Japan. Received January 18, 2005; accepted February 28, 2005
}

Seven sterols $(1-7)$ and eight polyisoprenepolyols $(8-15)$, isolated from the non-saponifiable lipid fraction of the dichloromethane extract of an edible mushroom, Hypsizigus marmoreus (Buna-shimeji), were tested for their antitubercular activity against Mycobacterium tuberculosis strain $\mathrm{H}_{37} \mathrm{Rv}$ using the Microplate Alamar Blue Assay (MABA). Six sterols $(2-7)$ and two polyisoprenepolyols $(8,12)$ showed a minimum inhibitory concentration (MIC) in the range of $1-51 \mu \mathrm{g} / \mathrm{ml}$, while the others $(1,9-11,13-15)$ were inactive $(\mathrm{MIC}>128 \mu \mathrm{g} / \mathrm{ml})$. The seven sterols $(1-7)$ and three polyisoprenepolyols $(8,10,12)$ were further evaluated for their inhibitory effects on Epstein-Barr virus early antigen (EBV-EA) activation induced by the tumor promoter 12-O-tetradecanoylphorbol-13-acetate (TPA) in Raji cells. Sterols 6 and 7 showed potent inhibitory effects while preserving the high viability of Raji cells.

Key words Hypsizigus marmoreus; sterol; polyisoprenepolyol; antituberculosis; Epstein-Barr virus early antigen

Hypsizigus marmoreus (Buna-shimeji) has recently become a popular edible mushroom in Asia, and has been cultivated by culture methods such as bottle culture and bag-culture. It has been reported to exhibit many biological activities such as anti-tumor, ${ }^{1,2}$ antioxidant, ${ }^{1)}$ and antiproliferative activity. ${ }^{3)}$ Several homologous polyisoprenepolyols named hypsiziprenols have recently been characterized in this mushroom. ${ }^{4)}$ Hypsiziprenols are the possible biosynthetic precursors of gymnopilins, bitter principles of $H$. marmoreus. ${ }^{4)}$ In the course of our studies on the bioactive principles of natural medicines and foodstuffs, ${ }^{5,6)}$ we evaluated the constituents of $H$. marmoreus for their antitubercular activity ${ }^{7)}$ as well as their inhibitory effects on Epstein-Barr virus early antigen (EBV-EA) activation induced by 12 - $O$-tetradecanoylphorbol-13-acetate (TPA). ${ }^{5,6)}$ In this paper, we describe the antitubercular activity against Mycobacterium tuberculosis of seven sterols (1-7) and eight polyisoprenepolyols, hypsiziprenols (8-15), and inhibitory effects on the induction of EBV-EA by the TPA of seven sterols $(\mathbf{1}-\mathbf{7})$ and three hypsiziprenols $(\mathbf{8}, \mathbf{1 0}, \mathbf{1 2})$ isolated from the non-saponifiable lipid (NSL) fraction of the extract of H. marmoreus.

\section{MATERIALS AND METHODS}

Extraction, Isolation, and Identification Sliced fresh bodies of Hypsizigus marmoreus $(11 \mathrm{~kg})$, cultivated in Nagano Prefecture in 2001, were air-dried. The dried mushroom $(833 \mathrm{~g})$ was then extracted with hot $\mathrm{CH}_{2} \mathrm{Cl}_{2}$ to give an extract $(86 \mathrm{~g})$. Alkaline hydrolysis $(5 \% \mathrm{KOH}$ in methanol $(\mathrm{MeOH})$, reflux, $3 \mathrm{~h})$ of the extract yielded a neutral nonsaponifiable lipid (NSL) fraction $(9.0 \mathrm{~g})$. Crystallization of the NSL fraction from $\mathrm{MeOH}$ yielded a precipitate $(2.8 \mathrm{~g})$, which was identified as ergosterol $(1),{ }^{2)}$ and a filtrate $(4.9 \mathrm{~g})$.
The filtrate was subjected to column chromatography on silica gel $(150 \mathrm{~g})$ using an $n$-hexane-ethyl acetate (EtOAc) gradient of $1: 0-1: 1$ as an eluent, which yielded fractions containing monohydroxy sterols (fraction $\mathrm{A} ; R f 0.83$ on TLC developed with EtOAc-MeOH, 4:1), polyhydroxy sterols (fraction $\mathrm{B} ; R f 0.48$ ), and polyisoprenepolyols (fraction $\mathrm{C} ; R f$ $0.24)$. Fractions $A-C$ were purified by further chromatography on silica gel. Reversed-phase preparative HPLC (HPLC I; $25 \mathrm{~cm} \times 10 \mathrm{~mm}$ i.d., Pegasil ODS II (Senshu Scientific Co., Ltd., Tokyo, Japan) column; mobile phase: $\mathrm{MeOH}-\mathrm{H}_{2} \mathrm{O}-$

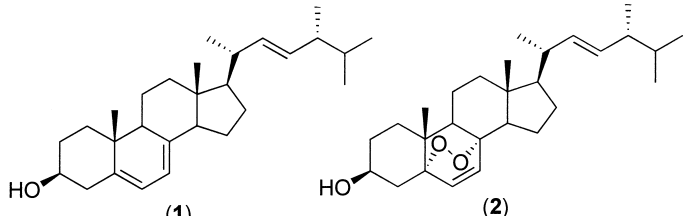

(1)

(2)

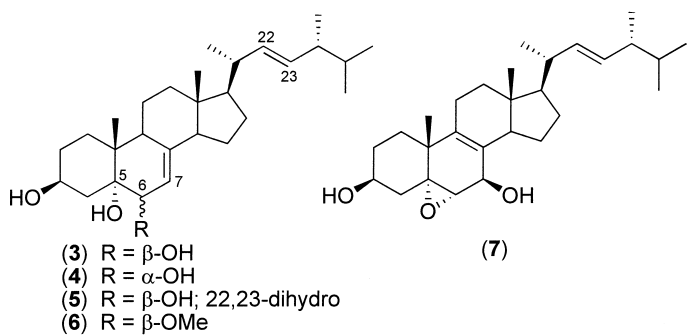

6) $\mathrm{R}=\beta-\mathrm{OMe}$

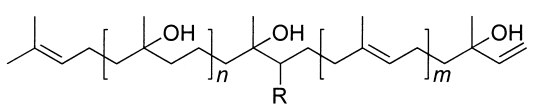

(8) $m=1, n=5, \mathrm{R}=\mathrm{H} \quad$ (12) $m=2, n=5, \mathrm{R}=\mathrm{OH}$

$\begin{array}{lll}\text { (9) } m=2, n=4, \mathrm{R}=\mathrm{H} & \text { (13) } m=1, n=7, \mathrm{R}=\mathrm{H}\end{array}$

$\begin{array}{ll}\text { (10) } m=1, n=5, \mathrm{R}=\mathrm{OH} & \text { (14) } m=1, n=8, \mathrm{R}=\mathrm{H} \\ \text { (11) } m=2, n=5, \mathrm{R}=\mathrm{H} & \text { (15) } m=1, n=8, \mathrm{R}=\mathrm{OH}\end{array}$

Chart 1. Structures of Compounds $\mathbf{1}-\mathbf{1 5}$ 
acetic acid $(\mathrm{AcOH}), 19: 1: 1,2.0 \mathrm{ml} / \mathrm{min})$ of purified fraction A $(0.39 \mathrm{~g})$ gave compound $\mathbf{1}\left(60 \mathrm{mg}\right.$; retention time $\left(t_{\mathrm{R}}\right)$ $28.3 \mathrm{~min}$ ) and ergosterol peroxide $(2)^{2)}\left(12 \mathrm{mg} ; t_{\mathrm{R}} 27.8 \mathrm{~min}\right)$. HPLC (HPLC II; column used was the same as that of HPLC I; mobile phase: $\mathrm{MeOH}-\mathrm{H}_{2} \mathrm{O}-\mathrm{AcOH}, 9: 1: 1,2.0 \mathrm{ml} / \mathrm{min}$ ) of purified fraction $\mathrm{B}(0.38 \mathrm{~g})$ gave five compounds, cerevisterol $(3)^{8)}\left(40 \mathrm{mg} ; t_{\mathrm{R}} 23.6 \mathrm{~min}\right), 6$-epicerevisterol (4) $)^{9)}\left(1.7 \mathrm{mg} ; t_{\mathrm{R}}\right.$ $38.6 \mathrm{~min}), \quad 22,23$-dihydrocerevisterol $\quad(5)^{10)} \quad\left(6.7 \mathrm{mg} ; \quad t_{\mathrm{R}}\right.$ $28.6 \mathrm{~min}), \quad 6-O$-methylcerevisterol $\quad(6)^{11)} \quad\left(2.4 \mathrm{mg} ; \quad t_{\mathrm{R}}\right.$ $37.1 \mathrm{~min})$, and $(22 E, 24 R)-5 \alpha, 6 \alpha$-epoxyergosta-8,22-diene$3 \beta, 7 \beta$-diol (7) ${ }^{12)}\left(5.0 \mathrm{mg} ; t_{\mathrm{R}} 21.7 \mathrm{~min}\right)$. HPLC (HPLC III; $25 \mathrm{~cm} \times 10 \mathrm{~mm}$ i.d., Hypersil ODS-5 (Senshu Scientific Co., Ltd., Tokyo, Japan) column; mobile phase: $\mathrm{MeOH}-\mathrm{H}_{2} \mathrm{O}-$ $\mathrm{AcOH}, 75: 25: 1,0.8 \mathrm{ml} / \mathrm{min})$ of purified fraction $\mathrm{C}(1.28 \mathrm{~g})$ gave eight hypsiziprenols, ${ }^{4)}$ hypsiziprenol $\mathrm{A}_{9}(\mathbf{8})\left(77 \mathrm{mg} ; t_{\mathrm{R}}\right.$ $24.8 \mathrm{~min} ; \mathrm{mp} 30-31^{\circ} \mathrm{C}$ after crystallization from $\mathrm{MeOH}$; $[\alpha]_{\mathrm{D}}^{25}+5.3^{\circ}(c=0.10$, acetone $)$; positive FAB-MS $m / z 739$ $\left.[\mathrm{M}+\mathrm{H}]^{+}\right)$, hypsiziprenol $\mathrm{B}_{9}(9)\left(1.1 \mathrm{mg} ; t_{\mathrm{R}} 23.3 \mathrm{~min} ; \mathrm{mp}\right.$ $34-35^{\circ} \mathrm{C} ;[\alpha]_{\mathrm{D}}^{25}+6.2^{\circ}(c=0.10$, acetone $)$; positive FAB-MS $\left.m / z \quad 721[\mathrm{M}+\mathrm{H}]^{+}\right)$, hypsiziprenol $\mathrm{AA}_{9}$ (10) $\left(2.3 \mathrm{mg} ; t_{\mathrm{R}}\right.$ $20.6 \mathrm{~min} ; \mathrm{mp} 65-67^{\circ} \mathrm{C} ;[\alpha]_{\mathrm{D}}^{25}+2.8^{\circ} \quad(c=0.11$, acetone $)$; negative FAB-MS $\left.m / z 753[\mathrm{M}-\mathrm{H}]^{-}\right)$, hypsiziprenol $\mathrm{B}_{10}(\mathbf{1 1})$ $\left(2.0 \mathrm{mg} ; t_{\mathrm{R}} 18.8 \mathrm{~min} ; \mathrm{mp} 45-47^{\circ} \mathrm{C} ;[\alpha]_{\mathrm{D}}^{25}+8.5^{\circ}(c=0.10\right.$, acetone); positive FAB-MS $m / z 807[\mathrm{M}+\mathrm{H}]^{+}$), hypsiziprenol $\mathrm{BA}_{10}(12)\left(4.3 \mathrm{mg} ; t_{\mathrm{R}} 21.8 \mathrm{~min} ; \mathrm{mp} 41-43^{\circ} \mathrm{C} ;[\alpha]_{\mathrm{D}}^{25}+5.0^{\circ}\right.$ $\left(c=0.11\right.$, acetone); negative FAB-MS $\left.m / z 821[\mathrm{M}-\mathrm{H}]^{-}\right)$, hypsiziprenol $\mathrm{A}_{11}(\mathbf{1 3})\left(1.6 \mathrm{mg} ; t_{\mathrm{R}} 29.0 \mathrm{~min}\right.$; $\mathrm{mp} 41-43{ }^{\circ} \mathrm{C}$; $[\alpha]_{\mathrm{D}}^{25}+1.7^{\circ} \quad(c=0.20$, acetone $)$, negative FAB-MS $m / z 823$ $\left.[\mathrm{M}-\mathrm{H}]^{-}\right)$, hypsiziprenol $\mathrm{A}_{12}(\mathbf{1 4})\left(14.8 \mathrm{mg} ; t_{\mathrm{R}} 26.0 \mathrm{~min} ; \mathrm{mp}\right.$ $51-53^{\circ} \mathrm{C} ;[\alpha]_{\mathrm{D}}^{25}+4.5^{\circ}(c=0.10$, acetone $)$; negative FAB-MS $\left.m / z 995[\mathrm{M}-\mathrm{H}]^{-}\right)$, and hypsiziprenol $\mathrm{AA}_{12}(\mathbf{1 5})\left(2.5 \mathrm{mg} ; t_{\mathrm{R}}\right.$ $33.2 \mathrm{~min} ; \mathrm{mp} 65-67^{\circ} \mathrm{C} ;[\alpha]_{\mathrm{D}}^{25}+2.8^{\circ} \quad(c=0.11$, acetone $)$; negative FAB-MS $m / z 1011[\mathrm{M}-\mathrm{H}]^{-}$).

Identification of sterols $\mathbf{1}-\mathbf{7}$ was done by ${ }^{1} \mathrm{H}-\mathrm{NMR}$ $(400 \mathrm{MHz})$ and EI-MS spectral comparison with the corresponding compounds in the literature, ${ }^{2,8-12)}$ and identifica- tion of hypsiziprenols $\mathbf{8}-\mathbf{1 5}$ by ${ }^{1} \mathrm{H}-\mathrm{NMR}$ and FAB-MS spectral comparison with the corresponding or relevant compounds in the literature. ${ }^{4)}$

Determination of Antitubercular Activity The antitubercular activity of compounds in DMSO was determined against Mycobacterium tuberculosis $\mathrm{H}_{37} \mathrm{Rv}$ (ATCC 27294) in Middlebrook 7H12 medium using the Microplate Alamar Blue Assay (MABA), as previously described. ${ }^{13)}$ The minimum inhibitory concentration (MIC) is defined as the lowest concentration effecting a reduction in fluorescence of $90 \%$ relative to controls.

EBV-EA Induction Tests The EBV genome-carrying lymphoblastoid cells, Raji cells, derived from Burkitt's lymphoma, were cultured in RPMI-1640 medium. The Raji cells were incubated for $48 \mathrm{~h}$ at $37^{\circ} \mathrm{C}$ in a medium containing $n$ butyric acid ( $4 \mathrm{~mm})$, TPA (32 pM), and various amounts of test compounds. Smears were made from the cell suspension, and the EBV-EA-inducing cells were stained by means of an indirect immunofluorescence technique. The details of the in vitro assay on EBV-EA induction have been reported previously. $^{14)}$

\section{RESULTS AND DISCUSSION}

Although a homologous series of hypsiziprenols have previously been characterized in Hypsizigus marmoreus, mainly with the aid of FAB-MS spectroscopy, ${ }^{4}$ this study constitutes the first instance of their isolation and full characterization.

The MIC values of seven sterols (1-7) and eight hypsiziprenols (8-15) against $M$. tuberculosis are shown in Table 1. Among the sterols tested, compounds $\mathbf{2}-\mathbf{7}$ exhibited activity with MIC values of $1-51 \mu \mathrm{g} / \mathrm{ml}$, among which ergosterol peroxide $(2 \text {; MIC } 1 \mu \mathrm{g} / \mathrm{ml})^{15}$ followed by cerevisterol $(3 ; 6 \mu \mathrm{g} / \mathrm{ml})$ and 6-epicerevisterol $(4 ; 11 \mu \mathrm{g} / \mathrm{ml})$ showed the most potent activity, although still being an order of magnitude less potent than the first-line antitubercular drug, ri-

Table 1. Minimum Inhibitory Concentrations (MIC Values) against Mycobacterium tuberculosis $\mathrm{H}_{37} \mathrm{Rv}$ and Percentage of Epstein-Barr Virus Early Antigen (EBV-EA) Induction of Compounds $\mathbf{1}-\mathbf{1 5}$

\begin{tabular}{|c|c|c|c|c|c|c|c|c|}
\hline & \multirow{3}{*}{ Compound } & \multirow{3}{*}{$\begin{array}{c}\begin{array}{c}\text { Antitubercular } \\
\text { activity }\end{array} \\
\text { MIC }(\mu \mathrm{g} / \mathrm{ml})\end{array}$} & \multicolumn{5}{|c|}{ Percentage of EBV-EA induction ${ }^{a)}$} & \multirow{3}{*}{$\begin{array}{c}\left.\mathrm{IC}_{50}{ }^{b}\right) \\
\text { (mol ratio/32 } \\
\text { pmol TPA) }\end{array}$} \\
\hline & & & \multicolumn{5}{|c|}{ Concentration (mol ratio/32 pmol TPA) } & \\
\hline & & & 100 & & 500 & 100 & 10 & \\
\hline 1 & Ergosterol & $>128^{15)}$ & 12.2 & $(70)$ & 49.6 & 82.3 & 100 & 520 \\
\hline 2 & Ergosterol peroxide & $1^{15)}$ & 14.5 & (70) & 51.8 & 83.6 & 100 & 525 \\
\hline 3 & Cerevisterol & 6 & 11.6 & (70) & 49.0 & 80.9 & 100 & 518 \\
\hline 4 & 6-Epicerevisterol & 11 & 8.5 & $(70)$ & 45.9 & 80.1 & 100 & 512 \\
\hline 5 & 22,23-Dihydrocerevisterol & 51 & 10.3 & $(70)$ & 48.0 & 80.2 & 100 & 515 \\
\hline 6 & 6-O-Methylcerevisterol & 36 & 0 & (70) & 26.4 & 72.0 & 91.2 & 298 \\
\hline 7 & $(22 E, 24 R)-5 \alpha, 6 \alpha$-Epoxyergosta- 8,22 -diene- $3 \beta, 7 \beta$-diol & 45 & 0 & $(70)$ & 23.1 & 70.0 & 89.3 & 192 \\
\hline 8 & Hypsiziprenol $\mathrm{A}_{9}$ & 15 & 48.6 & $(70)$ & 72.3 & 92.7 & 100 & 926 \\
\hline 9 & Hypsiziprenol $\mathrm{B}_{9}$ & $>128$ & & & & & & \\
\hline 10 & Hypsiziprenol $\mathrm{AA}_{9}$ & $>128$ & 13.5 & $(70)$ & 51.0 & 75.1 & 100 & 510 \\
\hline 11 & Hypsiziprenol $\mathrm{B}_{10}$ & $>128$ & & & & & & \\
\hline 12 & Hypsiziprenol $\mathrm{BA}_{10}$ & 44 & 12.1 & $(70)$ & 50.0 & 74.2 & 100 & 508 \\
\hline 13 & Hypsiziprenol $\mathrm{A}_{11}$ & $>128$ & & & & & & \\
\hline 14 & Hypsiziprenol $\mathrm{A}_{12}$ & $>128$ & & & & & & \\
\hline \multirow[t]{3}{*}{15} & Hypsiziprenol $\mathrm{AA}_{12}$ & $>128$ & & & & & & \\
\hline & Rifampin $^{c)}$ & 0.25 & & & & & & \\
\hline & $\beta$-Carotene $\mathrm{e}^{c)}$ & & 8.6 & $(70)$ & 34.2 & 82.1 & 100 & 397 \\
\hline
\end{tabular}

a) Values represent percentages relative to the positive control value. TPA $(3$ pmol, $20 \mathrm{ng})=100 \%$. Values in parentheses are viability percentages of Raji cells. $b$ ) $\mathrm{IC}_{50}$ represents the mol ratio to TPA that inhibits $50 \%$ of positive control $(100 \%)$ activated with 32 pmol TPA. $c$ ) Reference compound. 
fampin (Table 1). From the limited data set, it appears that $\mathrm{C}$ $6 \alpha, 8 \alpha$-epidioxidation (2), hydroxylation at both C-5 $\alpha$ and C$6 \alpha / \beta(3-6)$, or hydroxylation at $\mathrm{C}-7 \beta$ accompanied with $\mathrm{C}$ $5 \alpha, 6 \alpha$-epoxidation (7) gives rise to higher activity for the $3 \beta$-hydroxy sterols. On the other hand, two compounds, hypsizprenol $\mathrm{A}_{9}(\mathbf{8} ; \mathrm{MIC} 15 \mu \mathrm{g} / \mathrm{ml})$ and hypsiziprenol $\mathrm{BA}_{10}$ (12; $44 \mu \mathrm{g} / \mathrm{ml}$ ), showed potent antitubercular activity among the eight hypsiziprenols (8-15) tested. There was no apparent correlation between the structures and antitubercular activity for the hypsiziprenols.

The inhibitory effects of the seven sterols $(\mathbf{1}-\mathbf{7})$ and three hypsiziprenols $(\mathbf{8}, \mathbf{1 0}, \mathbf{1 2})$ on EBV-EA activation induced by TPA were examined for the primary screening of anti-tumor promoting activities, and the results are shown in Table 1. Sterols 6 ( $\mathrm{IC}_{50}$ value $\left.298 \mathrm{~mol} \mathrm{ratio} / 32 \mathrm{pmol} \mathrm{TPA}\right)$ and 7 (192 mol ratio/32 pmol TPA) showed potent inhibitory effects while preserving the high viability of Raji cells. The inhibitory effects of these sterols were more potent than $\beta$ carotene (397 mol ratio/32 pmol TPA), a vitamin A precursor that has been studied extensively in cancer chemoprevention using animal models. ${ }^{16)}$ The inhibitory effects against EBVEA activation have been demonstrated to be closely parallel to those against tumor promotion in vivo, ${ }^{5,6)}$ and the sterols 6 and 7 from $H$. marmoreus were therefore suggested to be valuable anti-tumor promoters (potential cancer chemopreventive agents). Although ergosterol (2) exhibited only a moderate inhibitory effect against EBV-EA activation (Table 1), this still might be of importance as a chemopreventive constituent of $H$. marmoreus since this constitutes the most predominant lipid constituent of $H$. marmoreus and has been demonstrated to inhibit the promotion of skin papillomas by TPA in 7,12-dimethylbenz $[a]$ anthracene (DMBA)-initiated mice. $^{2)}$

In summary, concerning with the lipid constituents of $H$. marmoreus, the sterols might be of importance from the points of view of antituberculosis and cancer chemoprevention, whereas the hypsiziprenols are considered to be of less importance. No hyspiziprenols from $H$. marmoreus tested have been reported to show anti-tumor activity on NCI-H292 or EL-4 cell lines. ${ }^{4)}$

Acknowledgements This work was supported in part by a grant from the Ministry of Education, Culture, Sports, Science, and Technology to promote multi-disciplinary research projects.

\section{REFERENCES}

1) Matsuzawa T., Saitoh H., Sano M., Tomita I., Ohkawa M., Ikekawa T., Yakugaku Zasshi, 118, 476-481 (1998)

2) Yasukawa K., Aoki T., Takido M., Ikekawa T., Saito H., Matsuzawa T., Phytother. Res., 8, 10-13 (1994).

3) Chang J. S., Son J. K., Li G., Oh E. J., Kim J. Y., Park S. H., Bae J. T., Kim H. J., Lee I. S., Kim O. M., Kozukue N., Han J. S., Hirose M., Lee K. R., Cancer Lett., 212, 7-14 (2004).

4) Sawabe A., Morita M., Kiso T., Kishine H., Ohtsubo Y., Ouchi S., Okamoto T., J. Agric. Food Chem., 47, 588-593 (1999).

5) Akihisa T., Yasukawa K., "Studies in Natural Products Chemistry, Vol. 25. Bioactive Natural Products (Part F)," ed. by Atta-ur-Rahman, Elsevier Science B.V., Amsterdam, 2001, pp. 43-87.

6) Akihisa T., Yasukawa K., Tokuda H., "Studies in Natural Products Chemistry, Vol. 29. Bioactive Natural Products (Part J)," ed. by Attaur-Rahman, Elsevier Science B.V., Amsterdam, 2003, pp. 73-126.

7) Akihisa T., Franzblau S. G., Ukiya M., Okuda H., Zhang F., Yasukawa K., Suzuki T., Kimura Y., Biol. Pharm. Bull., 28, 158-160 (2005).

8) Yaoita Y., Endo Y., Tani Y., Machida K., Amemiya K., Furumura K., Kikuchi M., Chem. Pharm. Bull., 47, 847-851 (1999).

9) Chen R. Y., Wang Y. H., Yu D. Q., Acta Botanica Sin., 33, 65-68 (1991).

10) Madaio A., Piccialli V., Sica D., J. Nat. Prod., 52, 952-961 (1989).

11) Kawagishi H., Katsumi R., Sazawa T., Mizuno T., Hagiwara T., Nakamura T., Phytochemistry, 27, 2777-2779 (1988).

12) Ishizuka T., Yaoita Y., Kikuchi M., Chem. Pharm. Bull., 45, 17561760 (1997).

13) Collins L. S., Franzblau S. G., Agents Chemother, 41, 1004-1009 (1997).

14) Takaishi Y., Ujita K., Tokuda H., Nishino H., Iwashima A., Fujita T., Cancer Lett., 65, 19-26 (1992).

15) Cantrell C. L., Rajab M. S., Franzblau S. G., Fronczek F. R., Fischer N. H., Planta Med., 65, $732-734$ (1999).

16) Murakami A., Ohigashi H., Koshimizu K., Biosci. Biotech. Biochem., 60, 1-8 (1999). 